\title{
Nonlinear Generalisation of Quantum Mechanics
}

\author{
Alireza Jamali* \\ Senior Researcher \\ Natural Philosophy Department, Hermite Foundation ${ }^{\dagger}$ \\ alireza.jamali.mp@gmail.com
}

November 26, 2021

\begin{abstract}
It is known since Madelung that the Schrödinger equation can be thought of as governing the evolution of an incompressible fluid, but the current theory fails to mathematically express this incompressibility in terms of the wavefunction without facing problem. In this paper after showing that the current definition of quantum-mechanical momentum as a linear operator is neither the most general nor a necessary result of the de Broglie hypothesis, a new definition is proposed that can yield both a meaningful mathematical condition for the incompressibility of the Madelung fluid, and nonlinear generalisations of Schrödinger and KleinGordon equations. The derived equations satisfy all conditions that are expected from a proper generalisation: simplification to their linear counterparts by a well-defined dynamical condition; Galilean and Lorentz invariance (respectively); and signifying only rays in the Hilbert space.
\end{abstract}

Keywords - foundations of quantum mechanics, nonlinear quantum mechanics, nonlinear Klein-Gordon equation

\section{Contents}

1 Introduction $\quad 2$

1.1 A brief history . . . . . . . . . . . . . . . 2

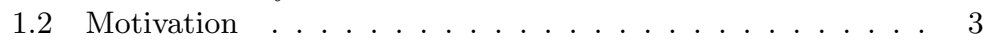

2 Harmonisation

* Corresponding author

$\dagger$ †rd Floor - Block No. 6 - Akbari Alley - After Dardasht Intersection - Janbazané Sharghi - Tehran - Iran 
3 Neoclassical Nonlinear theory 9

3.1 Generalisation of Schrödinger equation . . . . . . . . . . 9

3.1 .1 Galilean invariance . . . . . . . . . . . . . . 11

3.2 Generalisation of Klein-Gordon equation . . . . . . . . . . . 12

3.2.1 Lorentz invariance . . . . . . . . . . . . . . . 12

3.3 Comparison with other relevant nonlinear theories . . . . 13

4 Implications $\quad 14$

4.1 Generation of Probabilities . . . . . . . . . . . . . 15

4.2 Nonlinearity and Possibility of Solitons . . . . . . . . . 15

4.3 Commutativity . . . . . . . . . . . . . . . 15

5 Discussion

\section{Introduction}

\subsection{A brief history}

In the foundations of modern physics there is nothing more controversial than the measurement problem of quantum mechanics[1]: How should one reconcile the fact that on one hand, the wave function evolves deterministically according to the Schrödinger equation as a linear superposition of different states, while on the other hand, the outcome of measurements are always a single definite state?

This problem is a mere reappearance of the old issue of wave-particle duality, for as long as the wavefunction evolves one is dealing with a wave, while as soon as a measurement is performed one observes a single particle, a single dot on the screen for example. The reconciliation of the wave and particle pictures therefore is a crucial problem whose resolution is necessary for making any progress regarding the measurement problem. The pursuit of such reconciliation is at least as old as the quantum theory itself $^{1}$. It began with Mie's ideas[2] and was pursued seriously by Einstein after his successful explanation of the photoelectric effect[3]. Although in a letter to Besso[4] Einstein admitted that '[...] All the fifty years of conscious ruminations have not gotten me closer to an anwser for the question: "What are light quanta?" These days any rascal may believe that he knows, but he deludes himself.', his idea of particles being concentrations (lumps) of continuous fields[3][5] is still the best concrete idea we have for our attempts along such lines. This idea was a persistent theme of Einstein's attempts and was pursued through his grand programme of Unified Field Theory[5].

In fact Schrödinger himself initially thought in terms of wavepackets: a particle was assumed to be a 'parcel' of matterwaves (an envelope) that moved together as a whole by a group velocity equal to the velocity of the particle meanwhile the inner waves (in the parcel) oscillated by a frequency equal to the phase velocity of de Broglie's matterwaves. But this view soon faced a serious problem[6]: wavepackets which were supposed to be particles did not maintain their integrity due to linearity and

\footnotetext{
${ }^{1}$ We use 'quantum theory' to include also the so-called old quantum theory.
} 
dispersion ${ }^{2}$. This objection and the consequent 'victory' of the idealist founders like Bohr was serious enough to discourage even de Broglie for some twenty years [7]. de Broglie's interest in the problem was revitalised by Bohm's theory[8]; the theory that is now called de Broglie-Bohm or Pilot wave theory, according to which the particle and wave pictures are both maintained simultaneously and the wave 'guides' the particle[9] by the guiding equation

$$
\mathbf{p}=\nabla S
$$

where $S$ is the phase of the wavefunction (classically Hamilton's principal function $)[10]$. de Broglie-Bohm theory in its description of the doubleslit experiment, by considering the analogy between water waves and matterwaves[9], relies on the idea of the fluid behaviour of the wavefunction, which was proposed and elaborated by Madelung[11] shortly after Schrödinger's work. This fluid picture of Madelung will prove to be useful in our discussions in this paper.

de Broglie himself however considered pilot wave theory a 'degenerate' form of his early attempts[7]; such attempts evolved to what de Broglie in his late years dubbed as the theory of the double solution, according to which, particles were to be described by a nonlinear equation which has the Schrödinger equation as its approximation, hence the return of the Einsteinian theme. But neither Einstein nor de Broglie were able to derive any such equation satisfactorily. In this paper we derive an equation that

- Is a generalisation of Schrödinger equation: it simplifies to Schrödinger equation by a certain mathematical condition, hence fulfilling Einsteinde Broglie's maxim.

- Unlike the so-called Nonlinear Schrödinger equation[12] and the GhirardiRimini-Weber (GRW) theory[13], it contains no new arbitrary parameters.

- Is nonlinear and dispersive, hence allows for soliton solutions[14].

- Satisfies Weinberg's homogeneity property[15] according to which if $\psi$ is a solution then so must be $Z \psi$ for arbitrary complex number $Z$; in other words pure states must be represented by rays in the Hilbert space, not vectors.

Our focus in this paper is on the physical and conceptual aspects and we do not attempt at solving the proposed equation, neither do we enter techincal discussions regarding the existence and uniqueness of solutions. As the purpose of this paper is to scrutinise and revise the very foundations of quantum theory we hope that the mathematically-inclined reader indulges us patiently whenever a heuristic approach is pursued in lieu of abiding by the established formalism.

\subsection{Motivation}

According to the current understanding, Schrödinger equation governs the evolution of the wavefunction between measurements and measurements

\footnotetext{
${ }^{2}$ Nonlinearity and dispersion however solves this problem by allowing the existence of soliton solutions. This is one of our achievements in this paper.
} 
'reset' the probabilities. If we compare this statement with the issue of wave-particle duality, we realise that observation of particles violates unitarity and conservation of probability

$$
\frac{\partial|\psi|^{2}}{\partial t}+\nabla \cdot\left(|\psi|^{2} \frac{\nabla S}{m}\right)=0
$$

in other words we maintain that it is the conservation of probability that obstructs the application of Schrödinger equation to the collapse process. Hence any reconciliation of wave and particle pictures must go beyond the law of conservation of probability. It now temporarily serves us to recall Penrose's description of quantum mechanics[1] in terms of U-process ${ }^{3}$ and R-process ${ }^{4}$. According to Penrose the complete evolution of the quantum state must look like ${ }^{5}$

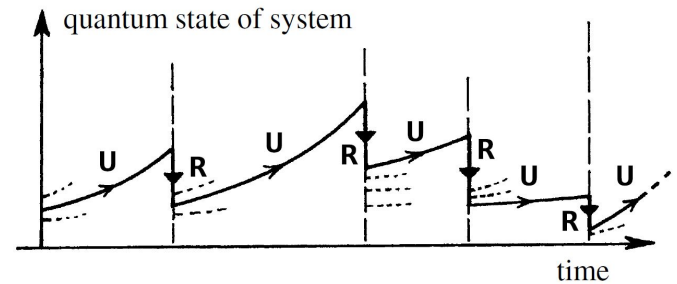

Figure 1: 'The way that the quantum-theoretic world appears to behave, with stretches of deterministic $\boldsymbol{U}$-evolution, punctuated by moments of probabilistic $\boldsymbol{R}$-action, each of which restores some element of classicality'[1].

If we recall the fluid picture of Madelung and hence think of the above graph as the motion of a quantum fluid of probability (hereafter Madelung fluid), we can presume that the $\boldsymbol{R}$-processes act as the sinks/sources of the Madelung fluid. But the current (orthodox) quantum mechanics crucially misses this point because the fluid that it describes is an incompressible one; in other words one without sinks/sources[16][17]. This condition in non-relativistic quantum mechanics is mathematically expressed by

$$
\nabla \cdot \mathbf{v}=\nabla \cdot \mathbf{p}=\nabla \cdot \mathbf{k}=0 .
$$

The conclusion is that a complete picture of reality in which a serious attempt is made for resolution of the wave-particle duality problem must dispose of the incompressibility of the Madelung fluid. It is an interesting fact that to the best of our knowledge relaxation of this significant restriction has not yet been considered.

Methodologically we know it is difficult to begin with the condition (2)

\footnotetext{
${ }^{3}$ Unitary evolution.

${ }^{4}$ Reduction (collapse of the wavefunction).

${ }^{5}$ The graph has a mere explanatory role and we do not consider it to accurately describe reality, for in our view there is not any such discontinuous $\mathbf{R}$-process.
} 
and arrive at a nonlinear equation. We must instead reverse the process and by starting from a firm physical motivation, find a nonlinear equation which simplifies to the Schrödinger equation by imposing (2).

Notwithstanding a key observation can be made immediately from (2), that the current scheme of quantum mechanics cannot handle the incompressibility condition (2) and in fact yields a contradiction: as

$$
\hat{\mathbf{p}}(\psi)=-i \hbar \nabla \psi
$$

condition (2) for a test wavefunction $\psi$ reads

$$
\nabla \cdot \hat{\mathbf{p}}(\psi)=-i \hbar \nabla^{2} \psi=0
$$

which is an absurdity since the derivation of (1) assumes $\nabla^{2} \psi \neq 0$ beforehand.

\section{Harmonisation}

It is the received wisdom that Planck-Einstein-de Broglie law ${ }^{6} p^{\nu}=\hbar k^{\nu}$ belongs to the era of 'old quantum mechanics' and that in the realm of quantum mechanics the right (and more fundamental) perspective is to solve the Schrödinger equation for any case at hand. Although from an instrumentalist point of view this perspective has been quite successful, in this paper we advocate another perspective which will prove to be more fruitful with regard to the foundational questions of quantum mechanics. Our perspective is that quantum mechanics is basically 'all' about $p^{\nu}=\hbar k^{\nu}$. To adopt such perspective we need to first scrutinise our understanding of its essential ingredient $k^{\nu}$. By any rigorous mathematical definition $^{7}$ it is required that a wave be defined as a field ${ }^{8}$ on spacetime which satisfies a certain equation, without any explicit reference to its four-wavevector. On the other hand, according to our perspective $p^{\nu}=\hbar k^{\nu}$ is a fundamental law of nature and appearance of $k^{\nu}$ in such a law suggests that we must enforce all waves to acquire a mathematically well-defined four-wavevector. Therefore we must find a definition for the four-wavevector of a wave $\psi$ in terms of the $\psi$ itself; we shall call this technique harmonisation. Considering the simplest case of a (complex)

\footnotetext{
${ }^{6}$ The metric signature $(+,-,-,-)$ is used everywhere in this paper. Greek indices run over 0 to 3 (four dimensions).

${ }^{7}$ For example, an entity $\phi$ which satisfies the wave equation

$$
\frac{\partial^{2} \phi}{\partial t^{2}}=v^{2} \nabla^{2} \phi,
$$

where $v$ is the speed of propagation of the wave. Or, an entity $\phi$ which satisfies the Schrödinger equation.

${ }^{8}$ We only consider scalar fields in this paper. Sufficient conditions of smoothness are also assumed implicitly.
} 
scalar harmonic wave w, $^{9,10}$,

$$
\psi=A e^{-i k_{\mu} x^{\mu}},
$$

if we apply the gradient operator to both sides we have,

$$
\partial_{\mu} \psi=-i k_{\mu} \psi=-i \psi k_{\mu}
$$

we realise that there are three possibilites for harmonisation:

1. Linear-operatorial approach, in which $\hat{k}_{\mu} \in \operatorname{End}\left(L^{2}(\mathbb{R})\right)^{11}$ i.e. fourwavevector is a linear operator (endomorphism) on the space of square-integrable functions, such that

$$
\psi \stackrel{\hat{k}_{\mu}}{\longmapsto} i \partial_{\mu} \psi
$$

i.e.

$$
\hat{k}_{\mu}(\psi):=i \partial_{\mu} \psi
$$

plus the eigenvalue hypothesis

$$
\hat{k}_{\mu}(\psi)=k_{\mu} \psi=\psi k_{\mu} .
$$

An immediate elaboration is needed here. Equation (4) is all that we have to begin with. It is valid only as it stands, and as it stands $k_{\mu}$ is a vector, not an operator on the $L^{2}$ space of wavefunctions. It is not impermissible to promote $k_{\mu}$ to an operator but to be logically consistent we cannot change the fundamental equation (4) that we have started with. Therefore if we choose to promote $k_{\mu}$ to an operator -as we do in this approach- we must keep (4) as it stands, and that is why we need the additional eigenvalue hypothesis. What we just stated is not anything controversial; it is one of the axioms[19] of quantum mechanics, only in light of a different perspective.

2. Nonlinear-operatorial ${ }^{12}$ approach, in which $\check{k}_{\mu}: L^{2}(\mathbb{R}) \backslash\{0\} \rightarrow L^{2}(\mathbb{R})$ such that

$$
\psi \stackrel{\check{k}_{\mu}}{\longmapsto} i \frac{\partial_{\mu} \psi}{\psi}
$$

i.e.

$$
\check{k}_{\mu}(\psi):=i \frac{\partial_{\mu} \psi}{\psi} \neq\left(k_{\mu} \psi=\psi k_{\mu}\right)
$$

without the need to add the eigenvalue hypothesis; because with the eigenvalue hypothesis in this case we would have

$$
i \frac{\partial_{\mu} \psi}{\psi}=k_{\mu} \psi
$$

\footnotetext{
${ }^{9}$ Notice that in these definitions only forward-in-time waves are considered. It is not clear whether this preference of time direction affects the theory in a decisive manner. Woit has elaborated on this issue in the context of QFT; see Appendix A to [18].

${ }^{10}$ Technically plane waves are not physically legitimate as they are not normalisable (squareintegrable), but this issue can be rigorously avoided; see[19]. We do not involve in such technical details hereafter in this paper.

${ }^{11}$ Again, technically quantum-mechanical operators are not defined on the entire $L^{2}(\mathbb{R})$ but only on a dense subspace. As we have already said, we do not involve in such technical details in this paper.

${ }^{12}$ We do not restrict our notion of 'operator' here to one which has the same domain and codomain.
} 


$$
\Rightarrow \partial_{\mu} \psi=-k_{\mu} \psi^{2}
$$

which is incompatible with (4).

3. Neoclassical nonlinear approach, in which $\psi \in L^{2}(\mathbb{R}) \backslash\{0\}$ and $k_{\mu}$ : $\mathbb{R}^{4} \rightarrow \mathbb{C}$ such that

$$
x^{\nu} \stackrel{k_{\mu}}{\longmapsto} i \frac{\partial_{\mu} \psi\left(x^{\nu}\right)}{\psi\left(x^{\nu}\right)}
$$

or

$$
k_{\mu}\left(x^{\nu}\right):=i \frac{\partial_{\mu} \psi\left(x^{\nu}\right)}{\psi\left(x^{\nu}\right)} \neq\left(k_{\mu} \psi=\psi k_{\mu}\right)
$$

again without the need to add the eigenvalue hypothesis, for the same reason stated above. As we shall see later the eigenvalue hypothesis is automatically included as a special case in this neoclassical approach in the sense that it leads to a generalisation of Schrödinger and Klein-Gordon eigenvalue problems (equations). The assumption $\psi \in L^{2}(\mathbb{R}) \backslash\{0\}$ is made only to make connection with the Born Principle, as our starting motivation requires. But after this (neoclassical) theory is developed it will become conceivable that the structure (Hilbert Space) that comes with this assumption might not be necessary in the neoclassical appraoch; especially the necessity of the inner product structure is hard to see given that in this approach -among other things- we do not need to define the notion of self-adjointness (which requires an inner product to be defined).

The second and third approach might seem identical but there are crucial differences. Notably in the nonlinear-operatorial approach -like linearoperatorial- $\psi$ and $\partial_{\mu} \psi$ are not considered 'independent'; once $\psi$ is given one just puts it into the input of $\breve{k}_{\mu}$ to get momentum. This is basically the reason that in the derivation of Schrödinger equation from the variational principle[20], one varies with respect to $\psi\left(\right.$ and $\left.\psi^{*}\right)$ but not $\partial_{\mu} \psi$. In the neoclassical approach however, $\psi$ and $\partial_{\mu} \psi$ are considered 'independent'. Indeed this is the reason for calling it neoclassical because it reminds one of the way position and momentum are treated in Hamiltonian mechanics.

The linear-operatorial approach is familiar and well-studied, being the foundation for orthodox quantum mechanics. The nonlinear-operatorial approach can handle the incompressibility condition, but in its current form is too special to yield a wave equation as it is now defined for a scalar field only. Hence the nonlinear-operatorial approach requires further consideration and a generalisation of the definition to be applicable to a vector field; we postpone full investigation of the nonlinear-operatorial approach however to another paper and in this paper focus on the neoclassical approach as it can both handle the incompressibility condition and yield a straightforward generalisation of Schrödinger and Klein-Gordon equations without the need to generalise the harmonisation technique to vector fields.

To the best of our knowledge our approach ${ }^{13}$ is new: it is the first time

\footnotetext{
${ }^{13}$ When we use 'our approach' we mean both the neoclassical and nonlinear-operatorial approaches. When a result or discussion is limited to the neoclassical approach and not true for the nonlinear-operatorial approach we specify it.
} 
that

$$
p_{\mu}=i \hbar \frac{\partial_{\mu} \psi}{\psi}
$$

is proposed as the definition of quantum-mechanical momentum. Although de Broglie-Bohm theory comes quite close to our approach, it misses the point by dispensing with the imaginary part of the momentum in order to make the same predictions as the Schrödinger picture. To be precise, if we write the wavefunction in polar form

$$
\psi(\mathbf{x}, t)=R(\mathbf{x}, t) e^{i S(\mathbf{x}, t) / \hbar}
$$

where $R, S: \mathbb{R}^{3} \times \mathbb{R} \rightarrow \mathbb{R}$, we see that according to our proposed definition of quantum-mechanical momentum (5) we have

$$
\mathbf{v}=\frac{\nabla S}{m}-\frac{i \hbar}{m} \frac{\nabla R}{R}
$$

while in de Broglie-Bohm theory only the first term is considered[8, 9, 21]; in our view there is no a priori theoretical reason why one should do so, on the contrary we will soon argue that neglecting the second term comes from a metaphysical ${ }^{14}$ position. By neglecting this second term one is losing some of the 'information' encoded in the wavefunction. This can be better seen if we think of $S$ as the Hamilton's principal function in classical mechanics (Hamilton-Jacobi theory[10]). We know that quantum mechanics must tell us more about reality than what classical mechanics does, however by leaving something unchanged (the guiding equation) that already exists in classical mechanics we cannot hope to fully achieve this expectation. Inclusion of the imaginary part is an important point of departure for our theory compared to de Broglie-Bohm theory, whose guiding equation is a special case of our definition when the second term

$$
\frac{i \hbar}{m} \frac{\nabla R}{R}
$$

is neglected. Orthodox quantum mechanics is not different with regard to this limitation because by its conservation of probability (1) it implicitly assumes $\mathbf{v}=\nabla S / m$. The basic reason that de Broglie-Bohm and orthodox quantum theory neglect the second term is that being bound by the eigenvalue hypothesis, they think of eigenvalues as what is actually observed in measurements, hence they require the eigenvalues to be real numbers, which is mathematically expressed by self-adjointness. We think that this is the last remnant of 'classical thinking' in quantum mechanics that presupposes only real numbers 'exist'. It is true that in measurements one only observes real numbers but that can well be a limitation of our understanding: that we cannot observe imaginary numbers is not a reason they cannot exist. This is why we stated earlier that the neglection of the imaginary part of the guiding equation stems from a metaphysical position $^{15}$. By relaxing this restriction, our approach yields novel insights

\footnotetext{
${ }^{14}$ By metaphysics we mean beyond physics.

${ }^{15}$ In fact our proposal of including the second term in (6) is quite aligned with the work of Renou et al.[22] who are discussing the possibility of empirically testing the 'reality' of complex numbers.
} 
which are obscured by narrowing physical entities to linear self-adjoint operators.

Even if we ignore this important point, on the conceptual side de BroglieBohm theory never promotes (5) to the definition of momentum in quantum mechanics as the theory only augments Schrödinger's theory with the so-claimed additional guiding equation.

Two aspects of imperfection of mathematics of quantum theory as it stands currently are therefore shown here. These imperfections provide a reason to think that definition (5) by utilising all the information encoded in the wavefunction, and by successfully handling the incompressibility condition as we shall see, is the 'right' definition ${ }^{16}$ for quantum-mechanical momentum by virtue of its generality.

\section{$3 \quad$ Neoclassical Nonlinear theory}

\subsection{Generalisation of Schrödinger equation}

We apply the Planck-Einstein-de Broglie law $p^{\mu}=\hbar k^{\mu}$ to our proposed definition (5) to get

$$
\mathbf{p}=-i \hbar \frac{\nabla \psi}{\psi} \quad \text { and } \quad E=\frac{i \hbar}{\psi} \frac{\partial \psi}{\partial t} .
$$

Similar to the familiar derivation of the Schrödinger equation from the law of conservation of energy, we expect to get the nonlinear equation for evolution of $\psi$ by substituting (7) in the law of conservation of energy

$$
E=\frac{p^{2}}{2 m}+V
$$

we must be cautious however, because just as in the case with derivation of Schrödinger equation, the square in the law of conservation of energy can be the source of a subtle confusion: if we think of quantum-mechanical p as a complex vector in $\mathbb{C}^{3}$,

$$
p^{2} \psi \stackrel{?}{=}\langle\mathbf{p}, \mathbf{p}\rangle \psi=\left(\mathbf{p} \cdot \mathbf{p}^{*}\right) \psi=\hbar^{2} \nabla^{2} \psi
$$

where dot is the Euclidean inner product ${ }^{17}$, we fail to achieve the result we want $\left(p^{2} \psi=-\hbar^{2} \nabla^{2} \psi\right)$ hence the Schrödinger equation. Therefore this is not the right way to think about quantum-mechanical $\mathbf{p}$, as we can get the intended minus only if we define

$$
p^{2} \psi:=\langle\mathbf{p}, \mathbf{p}\rangle \psi:=(\mathbf{p} \cdot \mathbf{p}) \psi=-\hbar^{2} \nabla^{2} \psi
$$

\footnotetext{
${ }^{16}$ Logically there is no right-wrong (true-false) binary system for definitions: a definition is only as good and general as the theory that is built upon it. Therefore a 'right definition' must be defined to be one which is more theoretically beneficial.

${ }^{17}$ There is a third possibility that

$$
p^{2} \psi \stackrel{?}{=}\langle\mathbf{p}, \mathbf{p} \psi\rangle=\mathbf{p} \cdot(\mathbf{p} \psi)^{*}=\hbar^{2} \nabla^{2} \psi^{*}
$$

which is of little interest.
} 
As the latter definition is the correct definition by yielding Schrödinger equation, any generalisation must conform to it. Consequently for our definition (7) we have

$$
p^{2}=\langle\mathbf{p}, \mathbf{p}\rangle=\mathbf{p} \cdot \mathbf{p}=-\frac{\hbar^{2}}{\psi^{2}} \nabla \psi \cdot \nabla \psi=:-\frac{\hbar^{2}}{\psi^{2}}\|\nabla \psi\|^{2} .
$$

Substitution in the law of conservation of energy now yields

$$
i \hbar \frac{\partial \psi}{\partial t}=-\frac{\hbar^{2}}{2 m} \frac{\|\nabla \psi\|^{2}}{\psi}+V \psi
$$

which can also be written as

$$
\frac{i \hbar}{2} \frac{\partial \psi^{2}}{\partial t}=-\frac{\hbar^{2}}{2 m}\|\nabla \psi\|^{2}+V \psi^{2} .
$$

Our equation (8) differs from the Schrödinger equation only by the term

$$
\frac{\|\nabla \psi\|^{2}}{\psi}
$$

which we now show only in the special case that $\mathbf{k}$ is a solenoidal field, is equal to the corresponding term in Schrödinger equation. Consider the incompressibility condition (2)

$$
\nabla \cdot \mathbf{k}=0
$$

which, by our definition (5) is

$$
\nabla \cdot\left(\frac{\nabla \psi}{\psi}\right)=\frac{\psi \nabla^{2} \psi-\|\nabla \psi\|^{2}}{\psi^{2}}=0
$$

therefore for an incompressible Madelung fluid one has

$$
\frac{\|\nabla \psi\|^{2}}{\psi}=\nabla^{2} \psi .
$$

In other words, Schrödinger equation is a special case of the nonlinear equation derived in this paper. Condition (11) reveals an important but hidden assumption of the current theory of quantum mechanics about wavefunctions; yet it is not unexpected at all, for it is equivalent -if we use the right definition of momentum- to the condition (2) which was already a well-known fact. What obscured this equation so far to be explicitly stated is the incomplete definition of momentum in orthodox quantum mechanics, which, as we remarked in the introduction, cannot handle the incompressibility condition satisfactorily.

We can now explicitly see how linearity of Schrödinger equation arises from nonlinearity of (8), and how an eigenvalue problem which is a marker of quantum discreteness and 'quantum jumps' is only a special case to a nonlinear but continuous reality. In this light the superposition 'principle' is but a special-case feature of nature and has a limited domain of applicability. 
The observation in which an eigenvalue problem arises from a more general nonlinear equation is quite a generic one and worthy of emphasis. As a simple example consider how Helmholtz equation

$$
\nabla^{2} \phi=-k^{2} \phi
$$

can be an approximation to the following nonlinear equation

$$
\nabla \cdot\left(\frac{\nabla \phi}{\phi}\right)=-k^{2}
$$

for $\phi \neq 0$, since

$$
\nabla \cdot\left(\frac{\nabla \phi}{\phi}\right)=\frac{1}{\phi} \nabla^{2} \phi-\frac{|\nabla \phi|^{2}}{\phi^{2}}=-k^{2}
$$

Multiplying both sides by $\phi$ yields

$$
\nabla^{2} \phi-\frac{|\nabla \phi|^{2}}{\phi}=-k^{2} \phi
$$

If we apply the approximation

$$
\frac{|\nabla \phi|^{2}}{\phi} \approx 0
$$

we are led to the original Helmholtz equation.

\subsubsection{Galilean invariance}

Equation (8) is Galilean-invariant in the same sense that Schrödinger equation itself is[20]: Assuming the gauge dependence of the wavefunction

$$
\psi^{\prime}\left(\mathbf{x}^{\prime}, t^{\prime}\right)=e^{-i\left(m \mathbf{v} \cdot \mathbf{x}-m v^{2} t / 2\right) / \hbar} \psi(\mathbf{x}, t),
$$

and the Galilean transformation of gradient and time derivatives

As

$$
\begin{gathered}
\nabla=\nabla^{\prime} \\
\frac{\partial}{\partial t}=\frac{\partial}{\partial t^{\prime}}-\mathbf{v} \cdot \nabla^{\prime}
\end{gathered}
$$

$$
\begin{gathered}
\frac{\partial \psi^{\prime}}{\partial t^{\prime}}=\frac{\partial \psi^{\prime}}{\partial t}+\mathbf{v} \cdot \nabla^{\prime} \psi^{\prime}=\frac{i}{2 \hbar} m v^{2} \psi^{\prime}+e^{-i\left(m \mathbf{v} \cdot \mathbf{x}-m v^{2} t / 2\right) / \hbar} \frac{\partial \psi}{\partial t}+\mathbf{v} \cdot \nabla \psi^{\prime} \\
=\frac{i}{2 \hbar} m v^{2} \psi^{\prime}+e^{-i\left(m \mathbf{v} \cdot \mathbf{x}-m v^{2} t / 2\right) / \hbar} \frac{\partial \psi}{\partial t}+\mathbf{v} \cdot\left(-\frac{i m}{\hbar} \mathbf{v} \psi^{\prime}+e^{-i\left(m \mathbf{v} \cdot \mathbf{x}-m v^{2} t / 2\right) / \hbar} \cdot \nabla \psi\right) \\
=e^{-i\left(m \mathbf{v} \cdot \mathbf{x}-m v^{2} t / 2\right) / \hbar}\left(-\frac{i}{2 \hbar} m v^{2} \psi+\frac{\partial \psi}{\partial t}+\mathbf{v} \cdot \nabla \psi\right),
\end{gathered}
$$

under this transformation the left-hand-side of our equation is

$$
i \hbar \frac{\partial \psi^{\prime}}{\partial t^{\prime}}=e^{-i\left(m \mathbf{v} \cdot \mathbf{x}-m v^{2} t / 2\right) / \hbar}\left(i \hbar \frac{\partial \psi}{\partial t}+\frac{1}{2} m v^{2} \psi+i \hbar \mathbf{v} \cdot \nabla \psi\right) .
$$


Since

$$
\begin{gathered}
\nabla^{\prime} \psi^{\prime}=\nabla \psi^{\prime}=e^{-i\left(m \mathbf{v} \cdot \mathbf{x}-m v^{2} t / 2\right) / \hbar}\left(-\frac{i m}{\hbar} \mathbf{v} \psi+\nabla \psi\right) \\
\left\|\nabla^{\prime} \psi^{\prime}\right\|^{2}=e^{-2 i\left(m \mathbf{v} \cdot \mathbf{x}-m v^{2} t / 2\right) / \hbar}\left(-\frac{m^{2}}{\hbar^{2}} v^{2} \psi^{2}-2 \frac{i m}{\hbar} \psi \mathbf{v} \cdot \nabla \psi+\|\nabla \psi\|^{2}\right),
\end{gathered}
$$

the right-hand-side is

$-\frac{\hbar^{2}}{2 m} \frac{\left\|\nabla^{\prime} \psi^{\prime}\right\|^{2}}{\psi^{\prime}}+V \psi^{\prime}=e^{-i\left(m \mathbf{v} \cdot \mathbf{x}-m v^{2} t / 2\right) / \hbar}\left(-\frac{\hbar^{2}}{2 m} \frac{\|\nabla \psi\|^{2}}{\psi}+V \psi+\frac{1}{2} m v^{2} \psi+i \hbar \mathbf{v} \cdot \nabla \psi\right)$

therefore

$$
i \hbar \frac{\partial \psi^{\prime}}{\partial t^{\prime}}=-\frac{\hbar^{2}}{2 m} \frac{\left\|\nabla^{\prime} \psi^{\prime}\right\|^{2}}{\psi^{\prime}}+V \psi^{\prime}
$$

\subsection{Generalisation of Klein-Gordon equation}

Our definition of momentum (5) can be readily substituted in $E^{2}=p^{2} c^{2}+$ $m^{2} c^{4}$ to yield

$$
\frac{1}{c^{2}}\left(\frac{\partial \psi}{\partial t}\right)^{2}-\|\nabla \psi\|^{2}+\left(\frac{m c}{\hbar}\right)^{2} \psi^{2}=0
$$

which can also be written as

$$
-\frac{\left\langle\partial_{\mu} \psi, \partial^{\mu} \psi\right\rangle}{\psi^{2}}=\left(\frac{m c}{\hbar}\right)^{2}
$$

Similar to the case for the non-relativistic equation (3), this equation is simplified to the Klein-Gordon equation as well via the special-relativistic generalisation of condition (2)

$$
\partial_{\mu} p^{\mu}=\partial_{\mu} k^{\mu}=0
$$

which is

$$
\begin{gathered}
\frac{\psi \square \psi-\left\langle\partial_{\mu} \psi, \partial^{\mu} \psi\right\rangle}{\psi^{2}}=0 \\
\Rightarrow \frac{\left\langle\partial_{\mu} \psi, \partial^{\mu} \psi\right\rangle}{\psi^{2}}=\frac{\square \psi}{\psi}
\end{gathered}
$$

Substitution in the alternative form (13), we have

$$
\frac{\square \psi}{\psi}=-\left(\frac{m c}{\hbar}\right)^{2}
$$

multiplication of both sides by $\psi$ yields

$$
\left(\square+\left(\frac{m c}{\hbar}\right)^{2}\right) \psi=0
$$

i.e. the Klein-Gordon equation.

\subsubsection{Lorentz invariance}

Equation (12) is Lorentz-invariant for the same reason that Klein-Gordon equation itself is. As our generalisation reads

$$
\left\langle\partial_{\mu} \psi, \partial^{\mu} \psi\right\rangle=-\psi^{2}\left(\frac{m c}{\hbar}\right)^{2},
$$

the right-hand-side being a scalar remains invariant under a Lorentz transformation; the left-hand-side is the inner product of Minkowski space which is also invariant under Lorentz transformations. 


\subsection{Comparison with other relevant nonlinear the- ories}

There are two existing main branches of research that involve nonlinearity in relation to quantum mechanics:

1. Particular Nonlinear Schrödinger equations; which are neither considered nor pursued as serious proposals for fundamental modifications of quantum mechanics.

2. General frameworks, like de Broglie's[7], GRW theory[13] and Weinberg's[23, $15]$; which pursue nonlinearity on a fundamental level, but involve arbitrariness.

Our proposal not only incorporates all the strengths of these two branches but also addresses their weaknesses: it is a general framework enjoying an explicit generalisation of Schrödinger and Klein-Gordon equations without any arbitrariness whatsoever.

The first category (particulars) includes the Nonlinear Schrödinger equation[12]

$$
i \frac{\partial \psi}{\partial t}=-\frac{1}{2 m} \nabla^{2} \psi+k|\psi|^{2} \psi
$$

Logarithmic Schrödinger equation[24]

$$
i \frac{\partial \psi}{\partial t}=-\frac{1}{2 m} \nabla^{2} \psi+b \psi \log |\psi|^{2},
$$

and other similar equations like Gross-Pitaevskii $[25]^{18}$. On the mathematical side, our proposed equation is totally different from such equations in that it is a first-order PDE. On the physical side, all such equations are based on various approximations[26][27] and with no simple derivation. Being approximations and not generalisations they all owe their physical role to a more fundamental (as opposed to emergent) equation. In case of the Gross-Pitaevskii equation for example, the nonlinearity is an emergent one $^{19}$ due to interactions between particles.

Our approach on the contrary is based on no approximation nor additional assumption or new parameters: it is based on the most general form of the de Broglie hypothesis that is possible to express in terms of the wavefunction itself using differential calculus. What totally distinguishes our proposed equation is that it is based on a firm physical foundation and simplifies to Schrödinger and Klein-Gordons equations without letting equal to zero any arbitrary parameter, but by imposition of a well-defined $d y$ namical condition that we already knew is assumed in quantum mechanics. For the reasons stated, the particular equations have not the potential of being extended to relativistic quantum mechanics while Special Relativity is easily applied to our proposed neoclassical definition of momentum (5) to yield the elegant generalisation of Klein-Gordon equation (12).

Along the lines of the second category that similar to our approach follow

\footnotetext{
${ }^{18}$ Weinberg[23] has a thorough review of such attempts and their status from the perspective of nonlinearity as a fundamental guiding principle.

${ }^{19}$ Involving scattering length $a_{s}$ which is not a fundamental constant of physics, the nonlinear term is due to interaction of particles and not a fundamental term present for single particles.
} 
nonlinearity as having a fundamental role in quantum physics, the term $\square \psi / \psi$ in (13) resembles $\square f / f$ in de Broglie's theory of double solutions[7]. Basically de Broglie considers two waves; usual $\psi$ and the $u$-wave. de Broglie thought of this new $u$-wave as representing a 'mobile singularity' intended to represent the particle aspect. The $f$ function is the amplitude of the $u$-wave. Although de Broglie himself -like the theory that bears his name- also missed the point by neglecting the new (second) term in (6), he did correctly realise the significance of $\square f / f$ by stating that 'The departure from the older mechanics is always bound up with the presence of the term $\square f / f$.' [7]. Apart from this remark, our theory is completely different from de Broglie's theory and does not appeal to the redundant notion of $u$-wave. More recent approaches include GRW theory[13], Weinberg's attempts[23][15] and Doebner-Goldin's[28]. As we mentioned earlier, GRW theory introduces two new parameters (collapse rate and localization distance) which are considered to be new fundamental constants of nature[29]. This is nothing but arbitrariness: new fundamental constants of nature can only be introduced out of absolute necessity; on the verge of desperation (Planck), otherwise we would have as many new 'fundamental' constants as there are trends of theoretical physics. By Occam's razor there is no need for new constants when we already have at hand a nonlinear fundamental theory without any arbitrariness. Same can be said about Doebner-Goldin equation[28]

$$
i \hbar \frac{\partial \psi}{\partial t}=-\frac{\hbar^{2}}{2 m} \nabla^{2} \psi+V \psi+i D \hbar\left(\frac{|\nabla \psi|^{2}}{|\psi|^{2}} \psi+\nabla^{2} \psi\right)
$$

due to the presence of arbitrary parameter $D$. Regarding Weinberg's theory, we maintain that its most important physical aspect is the homogeneity property according to which if $\psi$ is a solution then so is $Z \psi$ for arbitrary complex constant $Z$. This condition is so crucial for Weinberg that his proposed equation for evolution of wavefunction is motivated so as to satisfy it. Indeed our equation does satisfy this condition: as our equation for $Z \psi$ reads

$\frac{i \hbar Z^{2}}{2} \frac{\partial \psi^{2}}{\partial t}=-\frac{\hbar^{2}}{2 m} \nabla(Z \psi) \cdot \nabla(Z \psi)+V Z^{2} \psi^{2}=-\frac{\hbar^{2}}{2 m} Z^{2}\|\nabla \psi\|^{2}+V Z^{2} \psi^{2}$

it satisfies the condition for $\operatorname{arbitrary}^{20} Z$.

\section{Implications}

It must have become clear by now that our proposal is neither an alternative nor an interpretation of any existing quantum theory. It is a generalisation and as such it has novel physical consequences.

\footnotetext{
${ }^{20}$ Note that in the system that we are confined to (neoclassical approach), zero complex numbers are already excluded right from the beginning, so 'arbitrary' implicitly means nonzero arbitrary.
} 


\subsection{Generation of Probabilities}

Although our physical motivation was based on probabilities and measurements, after the development of our proposal we now face difficulties in maintaining such concepts. Our proposal cannot say anything about the Born Principle as its very statement that ${ }^{\prime}|\psi|^{2}$ is the probability distribution of a superposed quantum state switching to a single definite eigenvalue by performance of a measurement' is blurred by our proposal: according to our view there are neither superpositions nor eigenvalues in general; both are too special cases to deserve reference of a fundamental principle of nature. Possible redundancy of concepts like superposition and eigenvalues in turn makes the meaning of 'measurement' and 'probability of outcome of a measurement' unclear. In this light therefore, it is expected that we must look for a new meaning for $\psi$. In this paper however we follow Newton's maxim of hypotheses non fingo and leave the question open to further meticulous investigations. Accordingly we temporarily assume that $|\psi|^{2}$ is some sort of probability in order to demonstrate the following consequence. If we leave (almost) intact the assumption of $\rho=|\psi|^{2}$ representing probabilities, comparison of (1) and (6) implies that the rate of generation of probabilities denoted by $\pi$, is given by

$$
\pi=\left|\frac{i \hbar}{m} \nabla \cdot\left(|\psi|^{2} \frac{\nabla R}{R}\right)\right|
$$

Naturally $\pi=0$ is the situation in which probability is not generated and destructed i.e. conserved.

\subsection{Nonlinearity and Possibility of Solitons}

Notice that equation (8) is dispersive: Consider for example the case for a free particle

$$
i \frac{1}{\psi} \frac{\partial \psi}{\partial t}=-\frac{1}{2} \frac{\|\nabla \psi\|^{2}}{\psi^{2}}
$$

in which we have set $\hbar=m=1$ for simplicity. By our definition (5) the above equation yields the dispersion relation

$$
\omega=-\frac{1}{2}|\mathbf{k}|^{2},
$$

which is in fact identical with the dispersion relation that Schrödinger equation yields for a free particle. Unlike Schrödinger equation however, our equation is nonlinear. As we mentioned in the introduction it is known that dispersion and nonlinearity together allow for the possibility of existence of solitons[14]. It is therefore possible to revive the old notion of wavepackets as particles should such solutions actually occur.

\subsection{Commutativity}

The fundamental conceptual point of departure for quantum mechanics (in the Heisenberg picture) compared to classical mechanics is the canonical commutation relation

$$
\left[\hat{q}_{i}, \hat{p}_{j}\right]=i \hbar \delta_{i j}
$$


where $\hat{q}_{i}, \hat{p}_{j} \in \operatorname{End}\left(L^{2}\right)$. This fundamental relation states the operator algebra of quantum mechanics is not commutative.

According to the neoclassical approach however $q_{i}, p_{j}: \mathbb{R}^{4} \rightarrow \mathbb{C}$ i.e. momentum and position of a certain point in phase space are elements of the field of complex numbers (scalars) and by the commutative property of a field they do commute, viz. in the neoclassical approach (for simplicity we restrict to one dimension)

$$
p_{x}=-\frac{i \hbar}{\psi} \frac{\partial \psi}{\partial x}
$$

and

therefore

$$
x=-\frac{i \hbar}{\psi} \frac{\partial \psi}{\partial p_{x}}
$$

$$
p_{x} x=-\frac{i \hbar}{\psi} \frac{\partial \psi}{\partial x}\left(-\frac{i \hbar}{\psi} \frac{\partial \psi}{\partial p_{x}}\right)
$$

as long as all the constituents of this equation are elements of a field -which they are- we have

$$
-\frac{i \hbar x}{\psi} \frac{\partial \psi}{\partial x}=x p_{x}
$$

therefore in the neoclassical approach $x p_{x}=p_{x} x$.

It might be inferred from this observation that the Uncertainty Principle does not have a general validity as required by definition of a 'principle', but we prefer not to make any ontological conclusion here and leave the matter open to further investigations.

\section{Discussion}

The theory presented in this paper is sure to reproduce all the results of the orthodox quantum mechanics, as it is reduced to orthodox quantum mechanics by imposing the limitation expressed by (2). As such the theory is not an 'alternative' but a generalisation, a completion of orthodox quantum mechanics, in the same way that special relativity is a completion of Newtonian mechanics. There is a common prejudice regarding any proposal that tries to scrutinise the foundations of quantum mechanics that labels any such attempt as 'an alternative', leading naturally to expectations of disagreement between such proposals and the orthodox quantum theory, while it is a sheer logical contradiction if a generalisation disagrees with its own special case. The reason for this prejudice can be traced to the use of the word 'alternative' in the context of foundations of quantum mechanics. In that context 'alternative' is closely associated with 'interpretations', for example Many-Worlds interpretation or Relational interpretation. Those are theories with the same mathematical content but different ontologies while it would be imprudent to consider theories with the same mathematics as 'alternatives'21. There is but one theory that is an alternative in the proper sense of the word: GRW theory,

\footnotetext{
${ }^{21}$ That does not mean the author conforms to Kant's maxim that 'In any special doctrine of nature there can be only as much proper science as there is mathematics therein' but that we are simply against the use of such a strong word 'alternative' for these interpretations.
} 
which contains new ontic elements that have no basis in the mathematics of orthodox quantm mechanics, for example the Localisation Operator. Nobody thinks of special relativity as 'an alternative' to Newtonian mechanics in the same sense that Newtonian mechanics is an alternative to Aristotelian mechanics; it is a completion (generalisation) of Newtonian mechanics. To use the same example of special relativity, we know it reduces to Newtonian mechanics by letting to zero a well-defined quantity, for example by $\mathcal{O}\left(v^{4}\right)=0$ for the kinetic energy of a particle. Accordingly we define a 'generalisation' by two requirements:

- Addition of no new ontic dynamical elements.

- Simplification to the former theory by imposition a well-defined condition whose ontic basis -according to the first requirement- already exists in the former theory.

The example from special relativity satisfies both requirements as the notion of (kinetic) energy already exists in Newtonian mechanics, so does our proposal: No new ontological entity is introduced; all notions of quantummechanical momentum as based on Planck-Einstein-de Broglie law, a 'quantum fluid of probability' and its incompressibility already exist in the orthodox quantum mechanics. In this sense GRW theory is not a generalisation of orthodox quantum mechanics while it is easy to see that even the definition of momentum in orthodox quantum mechanics is a special case of our proposed definition (5):

$$
p_{\mu}=i \hbar \frac{\partial_{\mu} \psi}{\psi}
$$

simplifies to the orthodox counterpart

$$
\hat{p}_{\mu} \psi=p_{\mu} \psi=i \hbar \partial_{\mu} \psi
$$

by imposition of a well-defined condition $\left(\hat{p}_{\mu} \psi=p_{\mu} \psi\right)$ whose ontic basis already exists in the former theory. Both the definition of momentum, and the Schrödinger equation are therefore special cases of our theory. Hence our theory and the GRW theory are not comparable, being in two different categories. We thus see that there is no question that our proposed theory reproduces the results of orthodox quantum mechanics in its own limited realm, i.e. where the incompressibility condition and the eigenvalue hypothesis are valid. What we have shown is that there is no logical necessity for these conditions, by explicitly stating a satisfactory theory that does not obey them. So far no empirical tests of universal validity of these conditions could be made in principle, because as Einstein remarked that 'It is the theory which decides what we can observe'[30], it is only possession of a generalised theory that can demarcate the established theory.

Now that it is assured that our proposal cannot have any disagreement with the orthodox theory, it is a legitimate question that what new information this theory is giving us for the important experiments of quantum mechanics like the double-slit experiment? A proper answer of this question depends on solving equation (8) which the author has not yet been able to solve analytically. In absence of an analytical solution the next 
best action is to analyse the equation numerically which will be done in a consequent paper.

\section{Acknowledgement}

This work could never pass academic irrationalities had it not been for the continuous encouragements of Dr. Pouria Mistani to whom I am greatly indebted. I wish to thank Dr. Christian Böhmer for helpful discussions over the past two years. I also thank my friend Dr. Mohamad Emami for his role in the early development of my ideas through sophisticated discussions.

\section{References}

[1] Roger Penrose. Fashion, Faith, and Fantasy in the New Physics of the Universe. Princeton University Press, 2017.

[2] Leo Corry. From mie's electromagnetic theory of matter to hilbert's unified foundations of physics. Studies in History and Philosophy of Modern Physics, 30(2):159-183, 1999.

[3] Michel Janssen and Christoph Lehner, editors. The Cambridge Companion to Einstein. Cambridge University Press, 2014.

[4] Martin J. Klein. The first phase of the bohr-einstein dialogue. Historical studies in the physical sciences, 7(10), 2004.

[5] H.F.M. Goenner. On the history of unified field theories. Living Reviews in Relativity, 7(10), 2004.

[6] Wolfgang Pauli. Wave Mechanics: Volume 5 of Pauli Lectures on Physics. Books on Physics, Dover Publications, 2000.

[7] Louis de Broglie. Non-Linear Wave Mechanics: a Causal Interpretation. Elsevier, 1960.

[8] David Bohm. A suggested interpretation of the quantum theory in terms of "hidden" variables. i. Physical Review, 85(166), 1952.

[9] Jean Bricmont. Making sense of quantum mechanics. Springer, 2018.

[10] Cornelius Lanczos. The Variational Principles of Mechanics. Books on Physics, Dover Publications, 1986.

[11] Erwin Madelung. Eine anschauliche Deutung der Gleichung von Schrödinger. (German). Naturwissenschaften, 14(45):1004, 1926.

[12] V.E. Zakharov and S.V. Manakov. On the complete integrability of a nonlinear schrödinger equation. Journal of Theoretical and Mathematical Physics, 19(3):551-559, 1974.

[13] G.C. Ghirardi, A. Rimini, and T. Weber. Unified dynamics for microscopic and macroscopic systems. Physical Review D, 32(4):470-491, 1986.

[14] P. G. Drazin and R. S. Johnson. Solitons: An Introduction. Cambridge University Press, 1989. 
[15] Steven Weinberg. Testing quantum mechanics. Annals of Physics, 149(2):336-386, 1989.

[16] J. J. Sakurai and Jim Napolitano. Modern Quantum Mechanics. Cambridge University Press, 2017.

[17] G. K. Batchelor. An Introduction to Fluid Dynamics. Cambridge University Press, 2000.

[18] Peter Woit. Euclidean spinors and twistor unification (preprint). arXiv:2104.05099, 2021.

[19] Brian C. Hall. Quantum Theory for Mathematicians. Springer-Verlag New York, 2013.

[20] Eugen Merzbacher. Quantum Mechanics (Third Edition). John Wiley \& sons, inc., 1998.

[21] Detlef Dürr and Stefan Teufel. Bohmian Mechanics. Springer-Verlag Berlin Heidelberg, 2009.

[22] Marc-Olivier Renou et al. Quantum physics needs complex numbers (preprint). arXiv:2101.10873, 2021.

[23] Steven Weinberg. Precision tests of quantum mechanics. Physical Review Letters, 62(485):551-559, 1989.

[24] Abner Shimony. Proposed neutron interferometer test of some nonlinear variants of wave mechanics. Physical Review A, 20(394), 1979.

[25] E. P. Gross. Structure of a quantized vortex in boson systems. Il Nuovo Cimento, 20(3):454-457, 1961.

[26] Gadi Fibich. The Nonlinear Schrödinger Equation. Springer International Publishing, 2015.

[27] Catherine Sulem and Pierre-Louis Sulem. The Nonlinear Schrödinger Equation. Springer-Verlag New York, 1999.

[28] H.-D. Doebner and Gerald A. Goldin. On a general nonlinear schrödinger equation admitting diffusion currents. Physics Letters A, 162(5):397-401, 1992.

[29] Giancarlo Ghirardi and Angelo Bassi. Collapse theories. The Stanford Encyclopedia of Philosophy, 2020.

[30] Werner Heisenberg. Physics and Beyond. Harper \& Row, New York, 1972. 